\title{
Complementação do debate sobre Saúde da Criança e do Adolescente Indígena
}

\author{
Update on health of indigenous children and \\ adolescents
}

Complementación sobre la salud de la infancia y la
adolescencia indígena

Flávia Aparecida SQUINCA ${ }^{1}$

Dario PALHARES ${ }^{2}$

Joanice Gonçalves dos SANTOS ${ }^{3}$

\section{RESUMO}

A população indígena que habita o Brasilé de quase 900 mil indivíduos, divididos entre 305 etnias. A preocupação com a saúde das crianças e adolescentes indígenas foi reforçada na $4^{\text {a }}$ Conferência Nacional de Saúde Indígena. O objetivo deste trabalho é atualizar o debate sobre saúde da criança e adolescente indígena. No que tange ao perfil nutricional, os dados são conflitantes em estabelecer um diagnóstico de desnutrição, pois existe marcada convergência dos trabalhos citados no sentido de mostrar baixa estatura, porém com a manutenção da proporcionalidade peso/estatura. Tanto fatores étnicos como nutricionais podem estar presentes: déficits estaturais até certo percentual poderiam ser considerados como de origem étnica; acima disso, já indicariam desnutrição em nível populacional. Metade dos novos casos de tuberculose entre os índios é diagnosticada na infância e na adolescência. Todos os estudos apontam para a necessidade do aperfeiçoamento da política e das ações de saúde direcionadas às especificidades socioculturais dos povos indígenas.

Palavras-chave: Saúde de Populações Indígenas; Criança; Adolescente; Nutrição da Criança; Tuberculose; Política de Saúde.

\section{ABSTRACT}

The indigenous population of Brazil is of almost 900 thousand people, divided into 305 ethnic groups. The preoccupation with the health of children and adolescents was reinforced at the $4^{\text {th }}$ National Conference of Indigenous Health. The aim of this work is to update the debate about

1 Mestre. Assistente Social do Hospital Universitário de Brasília e Preceptora do Programa Educacional Tutorial de Saúde Indígena da Universidade de Brasília. Contato:flavia.squinca@gmail.com

2 Doutor. Pediatra do Hospital Universitário de Brasília e Preceptor do Programa Educacional Tutorial de Saúde Indígena da Universidade de Brasília.

3 Graduanda indígena do Povo Atikum de Pernambuco, do curso de Medicina da Universidade de Brasília. Programa Educacional Tutorial de Saúde Indígena da Universidade de Brasília. 
health conditions of indigenous children and adolescents. Regarding the nutritional profile, the data are conflicting in diagnosing undernourishing, since there is markedly convergence in the studied articles in showing short stature, but with maintainance of the proportion between weight and height. Both ethnic and nutritional aspects may be present: up to a certain proportion, deficits in height can be considered as of ethnical origin; above this value, this would indicate undernourishment in a populational basis. Half of the new cases of tuberculosis among Brazilian Indians are diagnosed in childhood and adolescence. All the studies point out to the necessity of improvement of policies and health actions directed to the sociocultural specificities of the indigenous peoples.

Key words: Health of Indigenous Peoples; Child. Adolescent; Child Nutrition; Tuberculosis; Health Policy.

\section{RESUMEN}

La población indígena que habita en Brasil es casi 900.000 personas, divididas entre los 305 grupos étnicos. La preocupación por la salud de los niños, niñas y adolescentes indígenas se reforzó en la $4{ }^{\text {a }}$ Conferencia Nacional de Salud Indígena. El objetivo de este estudio es actualizar el debate sobre la salud de los niños y adolescentes indígenas. En cuanto al perfil nutricional, los datos son contradictorios en el establecimiento de un diagnóstico de la desnutrición, porque hay fuerte convergencia de los trabajos citados con el fin de mostrar estatura, pero con el mantenimiento de la proporcionalidad peso/altura. Ambos factores étnicos y nutricionales pueden estar presentes: los déficits de estatura a un determinado porcentaje podrían tener origen étnico, en vez de indicar la desnutrición en la población. La mitad de todos los nuevos casos de tuberculosis entre los indios se diagnostica en la infancia y la adolescencia. Todos los estudios apuntan a la necesidad de mejora de las medidas de política y de la salud dirigidas a los derechos socio-culturales específicos de los pueblos indígenas.

Palabras clave: Salud de Poblaciones Indígenas; Niño; Adolescente; Nutrición del Niño; Tuberculosis; Política de Salud.

\section{INTRODUÇÃO}

Foi realizado no Brasil o I Inquérito Nacional de Saúde e Nutrição dos Povos Indígenas ${ }^{1}$, um trabalho de saúde pública até então inédito do Brasil e incomum nos demais países do mundo. A população indígena que habita o Brasil é de quase 900 mil indivíduos, que falam cerca de 180 idiomas e divididos entre 305 etnias $^{1,2}$. O Inquérito Nacional abordou diversos problemas que acometem a saúde indígena como um todo, tanto doenças crônicas típicas do adulto como especificidades da criança.

Os povos indígenas são reconhecidos em suas especificidades étnicas e culturais na Constituição Federal, nas Leis Orgânicas da Saúde e, principalmente, na Política Nacional de Atenção à Saúde 
dos Povos Indígenas. Para a efetividade da proteção, promoção e recuperação da saúde dos povos indígenas são imprescindíveis ações, modelos e serviços em consonância com as necessidades biopsicossociais dos índios e que, consequentemente, visem à garantia dos direitos de cidadania.

Segundo a Política Nacional de Atenção à Saúde dos Povos Indígenas, as Secretarias Estaduais e Municipais de Saúde, em parceria com a Fundação Nacional de Saúde (Funasa) do Ministério da Saúde, tinham como uma das atribuições a implementação de ações de saúde indígena integradas aos programas especiais - imunização, saúde da mulher e da criança, vigilância nutricional, controle da tuberculose, malária, doenças sexualmente transmissíveis e Síndrome da Imunodeficiência Adquirida (Aids), entre outros - assim como aos serviços de vigilância epidemiológica e sanitária a cargo dos gestores estaduais e municipais do Sistema Único de Saúde (SUS). No entanto, no ano de 2010, o Ministério da Saúde criou a Secretaria Especial de Saúde Indígena (Sesai) para coordenar e gerenciar o Subsistema de Atenção à Saúde Indígena (SasiSUS) do SUS, ou seja, para substituir a Funasa nas atribuições de proteção, promoção e recuperação da saúde integral dos povos indígenas, bem com nas ações de educação em saúde ${ }^{3}$.

A preocupação com a saúde das crianças e adolescentes indígenas também foi pauta da proposta de $n^{\circ} 25$ da $3^{\text {a }}$ Conferência Nacional de Saúde Indígena, realizada em 2001, ao enfatizar a necessidade de se priorizar a criação de programas na estruturação dos Distritos e Casas de Saúde do Índio. Em 2006, na $4^{\text {a }}$ Conferência Nacional de Saúde Indígena foi ratificada a importância e a urgência de ações de saúde específicas para atender às crianças e adolescentes indígenas, por exemplo, o monitoramento do processo de crescimento e desenvolvimento para combater a desnutrição e outras doenças.

\section{METODOLOGIA}

O objetivo deste trabalho é complementar, em relação ao I Inquérito Nacional de Saúde Indígena, o debate sobre a saúde da criança e do adolescente indígena habitante do Brasil. Tratase de uma revisão descritiva que não esgota o assunto nem realiza meta-análise, tendo por base os artigos publicados na Scientific Eletronic Library Online (Scielo) a partir da conjunção dos descritores em inglês: health, indigenous, child. A escolha pela base Scielo deveu-se pelos fatos de indexar as principais revistas do Brasil e da América Latina, totalizando cerca de 1041 periódicos, e de possibilitar acesso livre aos textos. Foram localizados 67 artigos, dos quais foram excluídos os trabalhos que versavam unicamente sobre antropologia ou sociologia e também os trabalhos que não versassem sobre criança ou adolescente, restando 26 trabalhos, todos sendo analisados e incluídos. Uma classificação preliminar de conteúdo evidenciou a predominância de trabalhos sobre desnutrição e tuberculose, havendo estudos esporádicos sobre outras condições clínicoepidemiológicas. Assim, o presente trabalho traz uma atualização sobre três tópicos acerca da saúde da criança e do adolescente indígena: perfil nutricional, tuberculose e outros agravos à saúde. 


\section{Perfil nutricional}

O estado nutricional das crianças e adolescentes indígenas responde pela maior parte dos artigos encontrados (10 artigos). Existem estudos das etnias Terena (Mato Grosso do Sul) ${ }^{4}$, Pakaanóva/ Wari' (Alto Xingu, Rondônia) ${ }^{5}$, Kaiowá e Guarani (Mato Grosso do Sul) ${ }^{6}$, Kaigáng (Paraná e Rio Grande do Sul ${ }^{7,8.9}$, Suruí (na fronteira de Rondônia com Mato Grosso) ${ }^{10}$, Kamaiurá ${ }^{11}$ ou Kamayurá ${ }^{12}$ (Alto Xingu, Mato Grosso - com referência à Floresta Amazônica). Recentemente, foi realizado o I Inquérito Nacional de Saúde e Nutrição dos Povos Indígenas ${ }^{13}$, o qual se baseou em amostragem mais ampla dos grupos indígenas presentes no Brasil. A avaliação do estado nutricional desde a infância é de fundamental importância para se trabalhar as políticas públicas de saúde, uma vez que a desnutrição e a obesidade afetam o processo de crescimento e desenvolvimento e podem gerar sequelas irreversíveis para a fase adulta.

Para o diagnóstico nutricional, o método de avaliação mais utilizado é a comparação das medidas de uma criança frente a uma amostra populacional significativa. Os dados de estatura por idade, peso por idade e peso por estatura são usualmente confrontados aos gráficos do Anthropometric Standardization Manual, da Organização Mundial de Saúde (OMS), sendo expressas em termos do escore $z$, ou seja, de quantos desvios-padrão a medida individual se desvia da média populacional. Por esse critério, os indivíduos no escore menor ou igual a $-2 z$ (ou seja, inferiores a -2 desviospadrão da média) são considerados deficitários (desnutridos). Já a obesidade ou sobrepeso é uma classificação para indivíduos que se encontrem acima do escore $+2 z$ (ou seja, acima de dois desvios-padrão da média) de peso por estatura. Além das medidas de peso e estatura, outras medições podem ser feitas, como a estimativa da porcentagem de gordura corporal/massa magra por impedanciometria, medidas da prega cutânea, etc.

Nos estudos das crianças e adolescentes indígenas os dados são conflitantes em estabelecer um diagnóstico de desnutrição, pois existe marcada convergência dos trabalhos citados e do I Inquérito Nacional de Saúde Indígena no sentido de mostrar baixa estatura, porém com a manutenção da proporcionalidade peso/estatura.

O grupo étnico Kaigang apresenta mais de 30 mil pessoas, sendo o quinto grupo em população, vivendo em terras indígenas nos estados de São Paulo, Paraná, Santa Catarina e Rio Grande do $\mathrm{Sul}^{7,8,9}$. Os menores agrupamentos contam com cerca de 300 pessoas e o maior deles, seis mil. Esse grupo foi estudado por Kuhl et al (2009) ${ }^{7}$, Manegolla et al (2006) ${ }^{8}$ e Castro et al (2010)9 ${ }^{9}$ O déficit de estatura para idade variou entre $15 \%$ a $35 \%$, aproximadamente, mas o déficit de peso para idade variou de praticamente zero a $12 \%$, enquanto a relação peso para estatura mostrou obesidade em proporção inferior a $1 \%$ e desnutrição, no estudo de Manegolla et al ${ }^{8}$, de cerca de $4 \%$. Nesse grupo, alguns indivíduos mostraram-se até mais altos (mais de 2 desvios-padrão) em relação à média mundial.

Os Pakaanóvas apresentaram déficit estatural para idade de $45 \%$, déficit ponderal de $25 \%$ e, // Tempus, actas de saúde colet, Brasília, 8(2), 211-223, jun, 2014.

ISSN 1982-8829 
no quesito peso/estatura, menos de $2 \%{ }^{4}$. Nesse grupo, apenas $10 \%$ das crianças apresentou escore z maior ou igual a zero e nenhuma criança chegou a um escore z de +2 . Entre os Kaiowás e Guaranis, de 2.800 crianças, 137 foram aleatoriamente avaliadas ${ }^{6}$. Cerca de 35\% das crianças apresentavam déficit de estatura para idade, $18 \%$ de peso para idade e 2 crianças apresentaram escore $\mathrm{z}$ maior que +2 . Os Terenas apresentaram tendência semelhante, $16 \%$ de déficit estatural, $8 \%$ de déficit ponderal, mas apenas $1 \%$ de déficit de peso para estatura ${ }^{4}$.

A pesquisa de Sampei et al (2007) ${ }^{12}$ sobre os Kamayurás mediu não apenas o peso e a estatura, mas também estimou a gordura corporal pela circunferência do braço e prega cutânea. Esse grupo populacional do Xingu é pequeno, com cerca de 90 pessoas, sendo que 65 tinham de 10 a 19 anos, as quais foram avaliadas. A tendência de baixa estatura foi observada, mas a relação peso/estatura apontou ou normalidade ou sobrepeso. A medida da gordura mostrou massa magra e gordura em proporções normais, ou seja, o sobrepeso aparentemente decorria de maior musculatura, não de obesidade. O estudo de Fagundes et al (2002) ${ }^{13}$ também com crianças do Alto Xingu não levou em conta a idade, apenas a relação entre peso por estatura e estimativa da gordura corporal por bioimpedância. Verificaram desnutrição em $1,8 \%$ da amostra e obesidade em 3\%.

Orellana et al (2006) ${ }^{10}$ estudaram crianças Suruís e compararam com os resultados publicados em 1987, na mesma população. Em 1987, 46\% das crianças apresentavam déficit estatural, 31\% baixo peso e $3,4 \%$ de baixo peso para estatura. Em 2005, 26\% de baixa estatura, $8 \%$ de baixo peso e nenhum baixo peso para estatura.

Assim, quando se trata da avaliação nutricional de grupos étnicos isolados, os instrumentos atualmente validados mostram limitações consideráveis. $\mathrm{O}$ primeiro questionamento que fica não respondido é se o déficit estatural decorre de fatores étnicos ou de fatores nutricionais.

Os estudos apresentados mostram que tanto fatores étnicos como nutricionais podem estar presentes quando se considera a baixa estatura em comparação com os gráficos de referência. Falando a favor de uma questão nutricional, o estudo de Orellana et a $l^{10}$ mostra um decréscimo acentuado na proporção de crianças com déficit estatural em 20 anos, de 46\% para 26\%. Mas, falando a favor de uma questão étnica, o estudo de Sampei et al $l^{12}$ aponta para a ausência de marcadores de desnutrição a despeito da baixa estatura verificada.

Diante de déficit nutricional crônico, o crescimento em estatura tende a ser prejudicado. Déficits crônicos leves geram o padrão observado, de baixa estatura com proporcionalidade de peso para estatura. Para a resposta definitiva à questão, uma amostra populacional significativa deveria ser provida, desde o nascimento, de recursos alimentares significativos, para daí então ser possível avaliar se o déficit estatural é nutricional ou é étnico. O mais provável é que haja uma base étnica gerando um déficit estatural; talvez, como indicado pelos estudos, algo em torno de 10 a $15 \%$ da população. Esse dado basal é de suma importância para o diagnóstico nutricional de populações indígenas do Brasil: déficits estaturais até certo valor podem ser considerados como de origem 
étnica; acima de determinado patamar, já indicariam desnutrição em nível populacional.

Também, o fato de em todas as amostras haver manutenção da proporcionalidade entre peso e estatura indica que, se houver carência nutricional, é uma carência intermediária, ou seja, nem é a plenitude nutricional desejável, mas tampouco a fome extrema. Alguns grupos populacionais eram muito pequenos e isso também é um fator de viés de comparação: pequenos grupos podem produzir resultados discordantes dos gráficos populacionais, mas quanto maior é a população estudada, ocorre maior proximidade ao modelo estatístico da OMS. Realmente, se para as etnias indígenas compostas por poucas pessoas todas as medições tenderam a ficar abaixo do escore $+2 \mathrm{z}$, quando se estudaram grupos étnicos mais populosos, já foram encontradas crianças acima do escore $+2 \mathrm{z}, \mathrm{o}$ que é estatisticamente esperado. Mas, ainda assim, apesar desses indivíduos mais altos, a tendência de déficit estatural persiste em todas as populações indígenas estudadas.

Em comum, todos os trabalhos descrevem os grupos indígenas como marginalizados economicamente, vivendo de agricultura de subsistência e alimentando-se predominantemente de amiláceos, com acréscimo de hortaliças, frutas e leguminosas e com reduzido acesso a carnes. Tais artigos apontaram para a necessidade de monitoramento da saúde das crianças e adolescentes indígenas, por meio de estudos sobre antropometria, estado nutricional e composição corporal. Uma das publicações ainda destacou a importância de se programarem pesquisas com a utilização de dados primários, com delineamento transversal, de coorte e ensaios randomizados controlados para ampliar os conhecimentos nutricionais sobre a população indígena e, consequentemente, implantar políticas capazes de atender às especificidades desse grupo.

São também necessárias ações e programas desde o período gestacional - com pré-natal e parto adequados - e que, posteriormente, considerem o contexto sociocultural e os hábitos dos grupos étnicos das crianças e adolescentes indígenas. Ademais, torna-se imprescindível a mobilização de políticas e programas intersetoriais de saúde, assistência social e educação, assim como serviços de saúde especializados, ações de saneamento básico, de garantia à água potável encanada e descontaminação ambiental para prevenir e diminuir o risco de doenças e os índices de mortalidade.

O contexto de exclusão social, de discriminação e as condições socioeconômicas e ambientais precárias de vida dos indígenas foram mencionados como determinantes que influenciam negativamente a saúde dessa população. As atividades e ações de vigilância nutricional e da anemia, para além do monitoramento do crescimento e desenvolvimento da criança indígena, foram apontadas como fundamentais. Também foi enfatizada a urgência de pesquisas sobre a saúde materno-infantil que possam auxiliar a melhoria das condições de saúde e nutricional deste segmento.

As atividades e ações de vigilância nutricional e da anemia, para além do monitoramento do crescimento e desenvolvimento da criança indígena, foram apontadas como fundamentais. Também 
foi enfatizada a urgência de pesquisas sobre a saúde materno-infantil que possam auxiliar a melhoria das condições de saúde e nutricional desse segmento. Além disso, os estudos sobre os indígenas das etnias Kaingang e Terena ratificam as informações já presentes na literatura científica, registram que o maior número de crianças desnutridas estava relacionado à falta de escolaridade das mães - 71,6\% das mães indígenas de Kaingang, região do Paraná, possuíam quatro anos de estudo ou menos $^{4,7}$. Todavia, segundo dados do governo Federal nas terras indígenas, 67,7\% dos indígenas de 15 anos ou mais de idade são alfabetizados. Já a taxa de alfabetização para os indígenas residentes fora das terras é de $85,5 \%{ }^{1}$.

\section{Tuberculose}

A tuberculose é um dos agravos à saúde que mais provoca a mortalidade e morbidade na população indígena. Foram identificados estudos de tuberculose das etnias Panará (Pará) ${ }^{14}$, Oro Waran Xijein (Rondônia) ${ }^{15}$, Suruí (Rondônia e Mato Grosso) ${ }^{16}$, Karajá e Tapirapé (Mato Grosso e Tocantins) ${ }^{17}$ e indígenas que residem no estado do Mato Grosso do Sul ${ }^{18,19}$.

Os resultados dos artigos analisados convergem no sentido que 40 a $50 \%$ da incidência de tuberculose entre os indígenas ocorre em menores de $15 \operatorname{anos}^{14,15,16,17,18,19}$. Apesar de o Brasil ser um dos países que apresenta uma das mais altas incidências de tuberculose, esse agravo é associado a situações precárias de vida, de modo que o risco de tuberculose não é homogêneo na população, concentrando-se em grupos específicos, como população de rua, prisioneiros e habitantes de favelas. A infecção pelo vírus da imunodeficiência humana (HIV) predispõe à tuberculose, havendo uma correlação forte HIV/tuberculose a ponto de o diagnóstico de uma condição indicar o rastreamento da outra. No entanto, no que tange à população indígena, poucos casos são associados ao HIV, sendo quase a totalidade dos casos relacionada às condições gerais de habitação desses grupos: casas com ventilação e iluminação inadequadas, com alta densidade de moradores em regime de co-leito ${ }^{14,16}$.

Dois estudos sobre os hábitos de sono com crianças das etnias Terena (Mato Grosso do Sul) e Bororo (Mato Grosso) mostraram a predominância do co-leito ${ }^{20,21}$. Em consonância, os dois estudos descrevem que o hábito de dormir juntos na mesma cama ou no mesmo quarto é influenciado por fatores familiares, ambientais e, principalmente, culturais. A pesquisa com indígenas da etnia Bororo afirmou que todas as crianças com menos dois anos dormem com outra pessoa e entre as crianças de 2 e 10 anos a frequência é de $81,5 \%$. A quantidade de pessoas em um mesmo quarto também foi registrada - entre os Bororos a média é de 5,1 no grupo de crianças com menos de 2 anos e 3,9 no grupo de crianças maiores de dois anos. Entre os Terena também é típico que duas ou mais pessoas durmam juntas na mesma cama, em quarto com média de 5,1 membros da comunidade indígena.

No que tange à vacinação contra a tuberculose, a Bacillus Calmette-Guérin (BCG), nas crianças indígenas, essa atinge cobertura superior a $80 \%$ na maioria das áreas, algumas chegando mesmo 
a $(100 \%)^{17}$. Entretanto, a incidência de tuberculose na população indígena é superior à média brasileira. No Brasil, a incidência de tuberculose é de aproximadamente 38 casos por 100.000 habitantes, enquanto, entre os indígenas do Mato Grosso do Sul, chegou a 300 casos por 100.000 habitantes, tendo se estabilizado em 50 casos por 100.000 habitantes a partir do ano de $2010^{19}$.

Apesar de cerca da metade dos novos casos de tuberculose entre os índios ser diagnosticada na infância e na adolescência, o diagnóstico dessa doença é particularmente difícil abaixo dos 5 anos de idade ${ }^{15,18}$. Tipicamente, o diagnóstico de tuberculose envolve a suspeita clínica de tosse incessante por mais de três semanas associada ao encontro de bacilos álcool-ácido resistentes no escarro. Não apenas as crianças menores de 5 anos são inábeis em escarrar, como geralmente a densidade bacilar no escarro desse grupo etário tende a ser pequena, de modo que a detecção ao exame de escarro apresenta menor sensibilidade. A baciloscopia e a cultura de escarro identificam cerca de $75 \%$ dos adultos portadores de tuberculose, mas entre as crianças menores de 5 anos, menos de $20 \%$ apresentam bacilos no escarro, e a cultura do escarro é negativa em mais de $50 \%$ das vezes. Na criança, os sintomas são comuns a outras doenças, e os achados radiológicos costumam ser inespecíficos ${ }^{15,18,19}$. A forma pulmonar da tuberculose é predominante, respondendo por mais de $80 \%$ das notificações dos casos. E mesmo entre os pacientes com manifestações extrapulmonares, cerca de $40 \%$ são crianças $^{15,16,18,19}$.

Para padronizar e orientar o diagnóstico e o tratamento de tuberculose, o Ministério da Saúde apresenta desde 2002 uma tabela de pontuação de dados clínicos que leva em conta o quadro clínico, o teste tuberculínico e a radiografia de tórax ${ }^{15}$. A dificuldade de realização do teste tuberculínico e de radiografias nas terras indígenas pode fazer com que o diagnóstico de tuberculose seja superestimado com base exclusivamente no quadro clínico dos pacientes: entre os Suruís (Rondônia), entre 1991 e 2000, foi observada uma incidência de tuberculose de mais de 2.500 casos/100 mil habitantes, valor aparentemente irreal, pois nem na era pré-tratamento e pré-vacinação a tuberculose apresentava tamanha incidência ${ }^{18}$. No entanto, conforme observam Gava et al $(2013)^{15}$, mesmo que a incidência de tuberculose seja superestimada em algumas áreas indígenas, os dados como um todo mostram que a tuberculose é um problema de saúde pública entre os indígenas, particularmente entre as crianças.

As medidas de saúde pública nas terras indígenas devem se pautar pelo rastreamento ativo de novos casos, para a realização do tratamento adequado. Na cadeia epidemiológica, é o adulto bacilífero que transmite a tuberculose para a criança. Assim, diante de um caso suspeito de tuberculose, é preciso que toda a família seja investigada, mediante a realização de teste tuberculínico e radiografia de tórax, sendo os casos negativos reavaliados após seis meses ${ }^{14,17}$. Ademais, embora não se possa afirma a correlação direta entre a prevalência da tuberculose e da desnutrição, emerge como imprescindível a realização de ações contínuas que avaliem a saúde integral da população indígena desde o nascimento.

Os resultados dos estudos sobre tuberculose registram as iniquidades em saúde entre a // Tempus, actas de saúde colet, Brasília, 8(2), 211-223, jun, 2014. 
população indígena e não indígena, assim como mostram os inquéritos de outras doenças que acometem esse segmento. Torna-se fundamental considerar a natureza multifatorial da tuberculose, os determinantes sociais e as especificidades culturais para a efetivação de políticas públicas de prevenção e recuperação da saúde dos indígenas acometidos pela doença.

\section{Outros agravos à saúde}

Além da questão nutricional, foram encontrados outros artigos sobre saúde da criança e adolescente indígena: déficit de atenção e hiperatividade ${ }^{22}$, diabete melito tipo $1^{23}$, deficiência visual $^{24}$, suicídio $^{25}$, hipoacusia ${ }^{26}$, pneumonia ${ }^{27}$ e estrutura etária, natalidade e mortalidade ${ }^{28}$. Embora o I Inquérito Nacional de Saúde Indígena ${ }^{1}$ tenha mencionado a diarreia, as afecções respiratórias altas, a malária, as hepatites e o atraso vacinal como importantes problemas de saúde pública da criança e do adolescente indígena, não foram encontrados estudos especificamente voltados para esses problemas, de modo que, tendo agora o inquérito por base, é possível que futuramente surjam estudos sobre essas condições.

O estudo sobre déficit de atenção/hiperativa foi realizado com participantes da etnia Karajá (Amazônia); um caso de diabetes melito 1 foi descrito em uma criança Yanomami (norte do Brasil e Sul da Venezuela); as pesquisas sobre deficiência visual e hipoacusia foram realizadas com crianças indígenas das etnias Guarani e Kaiowá (Mato Grosso do Sul); o estudo sobre o suicídio utilizou dados secundários do grupo étnico Guarani/Kaiowá (Mato Grosso do Sul); a investigação sobre pneumonia analisou dados de crianças indígenas de diferentes etnias, embora mais da metade dos dados tenha sido da etnia dos Xavante (Mato Grosso) e, por fim, a análise sobre estrutura etária, natalidade e mortalidade teve como objeto os dados epidemiológicos do povo Xavante.

Com relação a problemas visuais, Sá \& Bruno (2012) ${ }^{24}$ diferenciam déficit visual de problemas de refração. Na população brasileira de escolares, uma prevalência de 30\% de problemas refrativos é estimada, enquanto entre os escolares Guaranis e Kaiowás menos de 10\% apresentavam problemas de refração. No entanto, em uma triagem nas escolas, os autores puderam identificar 68 de um total de 6.600 crianças com deficiência visual, tendo-as encaminhado para tratamento especializado. Esses dados são considerados altos quando comparados aos registros da Organização Mundial da Saúde ${ }^{29}$. Também entre os Guaranis e Kaiowás, Pícoli et al (2006) ${ }^{26}$ rastrearam hipoacusia mediante o teste de emissões otoacústicas em crianças menores que 5 anos e encontraram uma prevalência de $5 \%$ de crianças com hipoacusia, que necessitavam de tratamento especializado e também tinham necessidades especiais no âmbito escolar.

A pesquisa sobre transtorno de déficit de atenção/hiperatividade (TDAH) teve como objetivo identificar, a partir dos critérios do Diagnostic and Statistical Manual of Mental Disorders, Fourth Edition (DSM-IV), a existência de sintomas de TDAH entre crianças indígenas da etnia Karajá $^{21}$. Dos convidados, 53 pais de crianças com 7 a 16 anos e que apresentavam alguma queixa comportamental ou emocional participaram do estudo, isto é, foram submetidos a uma entrevista 
com um psiquiatra infantil no posto de saúde da aldeia. Constatou-se que $24,5 \%$ das crianças entre 7 e 16 anos possuem sintomas de TDAH, tornando-se assim uma preocupação latente para os pais de crianças e adolescentes desse grupo étnico ${ }^{22}$.

O suicídio tem despontado como um problema de saúde pública entre os Guaranis sulmatogrossenses. Segundo Grubits et al (2011) ${ }^{25}$, houve 410 suicídios entre 2000 e 2008 na região, não contando as tentativas frustradas de suicídio. Nesse povo, o perfil do suicida típico é jovem, menor de 29 anos, do sexo masculino e o método mais comum é o enforcamento, sendo poucos casos de envenenamento e somente dois casos de uso de arma de fogo. Os autores registram dois episódios de suicídio em crianças, uma de cinco, a outra de seis anos de idade.

Todavia, dados da etnia Xavante apontam para a elevada concentração dos óbitos registrados como tendo ocorrido na faixa etária inferior a 5 anos de idade (77\% dos registros) ${ }^{28}$. As principais causas de morte foram as doenças endócrinas, nutricionais e metabólicas (24\%), as do aparelho respiratório (20\%) e infecciosas e parasitárias (5,6\%). Os autores levantam que esse cenário decorre de desnutrição infantil e que também $34 \%$ dos óbitos foram por sintomas mal definidos, apontando que há fragilidades sensíveis nos sistemas de saúde que atendem aos Xavantes. Essa fragilidade nos sistemas de saúde também foi evidenciada no estudo de Portela et al (2005) ${ }^{27}$ com dados de crianças indígenas internadas por pneumonia no Hospital Universitário de Brasília (HUB) - se, por um lado, eram pacientes com quadros graves de pneumonia (o que motivou a transferência para serviços de maior porte), o tratamento de $97 \%$ desses pacientes foi baseado em antibióticos usuais, de relativo baixo custo, os quais poderiam ter sido utilizados nos serviços locais de atenção à saúde indígena, ou seja, sem a necessidade de recursos mais onerosos.

\section{CONSIDERAÇÕES FINAIS}

Todos os estudos apontam para a necessidade do aperfeiçoamento da política e das ações de saúde direcionada aos povos indígenas. Para tanto, considera-se essencial a execução de políticas públicas intersetoriais - saúde, educação, assistência social, habitacional, ambiental e outras articuladas e que atendam as especificidades etárias e étnicas dos povos indígenas. Além disso, recomenda-se novos estudos antropológicos, sociais e epidemiológicos para o aprofundamento dos conhecimentos que permeiam o processo saúde e doença dos grupos indígenas brasileiros e, consequentemente, para fomentar mudanças na assistência à saúde desse segmento com específicas necessidades socioculturais.

\section{REFERÊNCIAS BIBLIOGRÁFICAS}

1. Fundação Oswaldo Cruz. Inquérito Nacional de Saúde e Nutrição dos Povos Indígenas. Relatório Final. Fiocruz: Rio de Janeiro, 2009. Disponível em: http:/www.abrasco.org.br/grupos/ arquivos/2013-01-23\%2013:44:23.pdf.

2. Instituto Brasileiro de Geografia e Estatística - IBGE (Brasil). Brasil tem quase 900 // Tempus, actas de saúde colet, Brasília, 8(2), 211-223, jun, 2014.

ISSN 1982-8829 
mil índios de 305 etnias e 274 idiomas. Brasília (DF): Ministério do Planejamento, Orçamento e Gestão, 2010. Disponível em: http://www.brasil.gov.br/governo/2012/08/brasil-tem-quase-900-mil-indios-de305-etnias-e-274-idiomas.

3. Silva EM, Santos JG, Silva GM, Hoefel MGL, Merchán-Hamann E, Severo DO, et al. Projeto vidas paralelas indígena: revelando o povo ATIKUM de Pernambuco, Brasil. Revista Tempus Actas de Saúde Coletiva. 2012; 6(1): 37-47.

4. Ribas DLB, Sganzerla A, Zorzatto JR, Philippi ST. Nutrição e saúde infantil em uma comunidade indígena Teréna, Mato Grosso do Sul, Brasil. Caderno de Saúde Pública. 2001; 17(2): 323-331.

5. Escobar AL, Santos RV, Junior CEAC. Avaliação nutricional de crianças indígenas Pakaanóva (Wari'), Rondônia, Brasil. Revista Brasileira de Saúde Materno Infantil. 2003; 3(4): 457-461.

6. Pícoli RT, Carandina L, Ribas DLB. Saúde materno-infantil e nutrição de crianças Kaiowá e Guaraní, Área Indígena de Caarapó, Mato Grosso do Sul, Brasil. Caderno de Saúde Pública. 2006; 22(1): 223-227.

7. Kühl AM, Corso ACT, Leite MS, Bastos JL. Perfil nutricional e fatores associados à ocorrência de desnutrição entre crianças indígenas Kaingáng da Terra Indígena de Mangueirinha, Paraná, Brasil. Caderno de Saúde Pública. 2009; 25(2): 409-420.

8. Menegolla IA, Drachler ML, Rodrigues IH, Schwingel LR, Scapinello E, Pedroso MB, et al. Estado nutricional e fatores associados à estatura de crianças da Terra Indígena Guarita, Sul do Brasil. Caderno de Saúde Pública. 2006; 22(2): 395-406.

9. Castro TJ, Schuch I, Conde WL, Veiga J, Leite MS, Dutra CLC, et al. Estado nutricional dos indígenas Kaingáng matriculados em escolas indígenas do Estado do Rio Grande do Sul, Brasil. Caderno de Saúde Pública. 2010; 26(9): 1766-1776.

10. Orellana JDY, Junior CEAC, Lourenço AEP, Santos RV. Nutritional status and anemia in Suruí Indian children, Brazilian Amazon. Jornal de Pediatria. 2006; 82(5): 383-388.

11. Mondini L, Canó EM, Fagundes U, Lima EES, Rodrigues D, Baruzzi RG. Condições de nutrição em crianças Kamaiurá - povo indígena do Alto Xingu, Brasil Central. Revista Brasileira de Epidemiologia. 2007; 10(1): 39-47.

12. Sampei MA, Canó EN, Fagundes U, Lima EES, Rodrigues D, Sigulem DM, et al. Avaliação antropométrica de adolescentes Kamayurá, povo indígena do Alto Xingu, Brasil Central (2000-2001). Caderno de Saúde Pública. 2007; 23(6): 1443-1453.

13. Fagundes U, Oliva CAG, Fagundes-Neto U. Avaliação do estado nutricional das crianças índias do Alto Xingu. Jornal de Pediatria. 2002; 78(5): 383-388.

14. Baruzzi RG, Barros VL, Rodrigues D, Souza ALM. Saúde e doença em índios Panará (Kreen-Akarôre) após vinte e cinco anos de contato com o nosso mundo, com ênfase na ocorrência de tuberculose (Brasil Central). Caderno de Saúde Pública. 2001; 17(2): 407-412. 
15. Gava C, Malacarne J, Rios DPG, Sant'Anna CC, Camacho LAB, Basta PC. Tuberculosis in indigenous children in the Brazilian Amazon. Revista de Saúde Pública. 2013; 47(1): 77-85.

16. Basta PC, Rios DPG, Alves LCC, Sant'Anna CC, Junior CEAC. Estudo clínicoradiológico de crianças e adolescentes indígenas Suruí, Região Amazônica. Revista da Sociedade Brasileira de Medicina Tropical. 2010; 43(6): 719-722.

17. Amarante JM, Costa VLA, Silva FA. Sensibilidade tuberculínica e vacina BCG entre os Índios do Araguaia - MT / 1997. Boletim de Pneumologia Sanitária. 1999; 7(1): 79-86.

18. Marques AMC, Pompilio MA, Santos SC, Garnês SJA, Cunha RV. Tuberculose em indígenas menores de 15 anos, no Estado de Mato Grosso do Sul. Revista da Sociedade Brasileira de Medicina Tropical. 2010; 43(6): 700-704.

19. Santos SC, Marques AMC, Oliveira RL, Cunha RV. Diagnóstico da tuberculose em indígenas menores de quinze anos por meio de um sistema de pontuação em Mato Grosso do Sul. Jornal Brasileiro de Pneumologia. 2013; 39(1): 84-91.

20. Reimão R, Souza JCRP, Medeiros MM, Almirão RI. Sleep habits in native Brazilian Terena children in the state of Mato Grosso do Sul, Brazil. Arquivos de Neuropsiquiatria. 1998; 56(4): 703-707.

21. Reimão R, Souza JCRP, Gaudioso CEV. Sleep habits in native Brazilian Bororo children. Arquivos de Neuropsiquiatria. 1999; 57(1): 14-17.

22. Azevêdo PVB, Caixeta L, Andrade LHS, Bordin IA. Attention deficit/hyperactivity disorder symptoms in indigenous children from the Brazilian Amazon. Arquivos de Neuropsiquiatria. 2010; 68(4): 541-544.

23. Gabbay MAL, Bussad E, Persoli L, Volpini W, Dib SA. Diabetes mellitus in a Young Amazon Indian child. Sao Paulo Medical Journal. 2005; 123(2): 93-95.

24. Sá MA, Bruno MMG. Deficiência visual nas crianças indígenas em idade escolar das etnias Guarani e Kaiowá na Região da Grande Dourados/ MS: Um estudo sobre a incidência e as necessidades específicas e educacionais especiais. Revista Brasileira de Educação Especial. 2012; 18(4): 629-646.

25. Grubits S, Freire HBG, Noriega JAV. Suicídios de Jovens Guarani/Kaiowá de Mato Grosso do Sul, Brasil. Psicologia: Ciência e Profissão. 2011; 31 (3): 504-517.

26. Pícoli RP, Carandina L, Ribas DLB. Hypoacousis prevalence in Kaiowá and Guarani indigenous children. Revista Brasileira de Saúde Materno Infantil. 2006; (2): 169-174.

27. Portela MFP, Sant'anna CC, Campos Júnior D. Pneumonia em crianças e adolescentes indígenas internados em Brasília-DF, Brasil: estudo de casos. Pulmão RJ 2005; 14(4):283-288.

28. Souza LG, Santos RV, Junior CEAC. Estrutura etária, natalidade e mortalidade do povo indígena Xavante de Mato Grosso, Amazônia, Brasil. Ciência \& Saúde Coletiva. 2010; 15(Supl. 1): $1465-1473$. 
29. Organização Mundial da Saúde. Magnitude e causas da deficiência visual. Geneve: OMS, 2004.

30. Ministério da Saúde/ Fundação Nacional de Saúde (FUNASA) / Sociedade Brasileira de Pediatria (SBP). Manual de Atenção à Saúde da Criança Indígena Brasileira. MS, 2004. Disponível em: http://www.redeblh.fiocruz.br/media/mnl_criancas.pdf

Artigo apresentado em 15/11/13

Artigo aprovado em 13/05/14

Artigo publicado no sistema em 29/06/14 